\title{
EFFECT OF THE COOLING RATE DURING MELT SOLIDIFICATION ON THE STRUCTURE AND PROPERTIES OF WC-W $\mathbf{W}_{2} \mathrm{C}$
}

\author{
I. Yu. Trosnikova, ${ }^{1,4}$ P. I. Loboda, ${ }^{1}$ O. P. Karasevska, ${ }^{2}$ \\ and O. I. Bilyi ${ }^{3}$
}

UDC 621.762

\begin{abstract}
The paper examines the effect of cooling rate on the structure, phase composition, size of coherent scattering domains, and stress-strain state of relit commercially used to strengthen machine parts and mechanisms that perform under abrasive wear and high contact loads. Optical and scanning electron microscopy, quantitative X-ray diffraction, and electron microprobe analysis have been employed to reveal that higher cooling rates during melt solidification decrease the sizes of phase components in the $20 \mathrm{wt} \% \mathrm{WC}-80 \mathrm{wt} \% \mathrm{~W}_{2} \mathrm{C}$ alloy and increase compressive stresses in the $\mathrm{W}_{2} \mathrm{C}$ matrix phase and tensile stresses in WC inclusions, improving the hardness of relit (by 1.5 times).
\end{abstract}

Keywords: tungsten carbide, cooling rate, stresses, hardness, grain-boundary strengthening mechanism.

\section{INTRODUCTION}

Melt-solidified eutectic alloys, representing a matrix from one refractory compound reinforced with inclusions of another refractory compound, are among the most promising materials that can efficiently be used to protect metal and alloy parts and structures against abrasive wear [1]. Relit is a eutectic alloy of tungsten carbides $\mathrm{WC}-\mathrm{W}_{2} \mathrm{C}\left(78-80 \mathrm{wt} . \% \mathrm{~W}_{2} \mathrm{C}, 20-22 \mathrm{wt} . \% \mathrm{WC}\right)$, whose production process has been adequately studied [2]. The melting point of relit is $2735^{\circ} \mathrm{C}$.

The tungsten carbide $\mathrm{WC}-\mathrm{W}_{2} \mathrm{C}$ alloy is used as wear-resistant coating material for parts that perform under intensive abrasive wear and moderate impact loads, specifically for coating of drill bits, parts of blast furnace feeders, and metalworking tools [2].

High strength at boundaries between components (the hardest inclusions and the matrix) is a necessary condition for wear-resistant material to perform in a reliable manner. Note that hard inclusions in the material are crumbled out if the components have low adhesion strength; i.e., there is abrasive wear. The wear rate of two-phase materials is determined by the chemical composition or nature and grain size of their components, as well as by loading and temperature in the contact area. Hardness and strength are the main criteria for assessing the wear resistance. The greatest wear resistance is exhibited by the $\mathrm{WC}-\mathrm{W}_{2} \mathrm{C}$ alloy, but its main drawback is high brittleness [3]. One of the ways to improve the wear resistance of ceramic composites may be to increase the rate at which the

${ }^{1}$ National Technical University of Ukraine 'Kiev Polytechnic Institute', Kiev, Ukraine. ${ }^{2}$ Kurdyumov Institute for Metal Physics, National Academy of Sciences of Ukraine, Kiev, Ukraine. ${ }^{3}$ Paton Electric Welding Institute, National Academy of Sciences of Ukraine, Kiev. Ukraine.

${ }^{4}$ To whom correspondence should be addressed; e-mail: irina2510@ukr.net.

Translated from Poroshkovaya Metallurgiya, Vol. 52, No. 11-12 (494), pp. 80-87, 2013. Original article submitted April 10, 2013. 
eutectic alloy solidifies from melt through grain refinement and fiber reinforcement. The papers $[4,5]$ show that higher cooling rates significantly improve the hardness of relit, but the nature of this phenomenon is still to be understood.

Our objective is to examine how the cooling rate influences the structure, phase composition, size of coherent scattering domains, and stress-strain state of relit.

\section{Experimental Procedure}

Tungsten carbides $\mathrm{WC}-\mathrm{W}_{2} \mathrm{C}$ were produced by grinding the ingots smelted in a Tammann-type furnace at $3100^{\circ} \mathrm{C}$. The grains are primarily fragmental. Mechanical grinding leads to numerous cracks in grains, decreasing their strength.

The alloy formation process was also studied by melting of relit compacts by an electron beam and centrifugal atomization of cylindrical billets smelted in a Tammann furnace. The cooling rate was $10^{2}{ }^{\circ} \mathrm{C} / \mathrm{sec}$ during smelting in a Tammann furnace and $10^{3}{ }^{\circ} \mathrm{C} / \mathrm{sec}$ during electron-beam melting. In the centrifugal atomization process, the cooling rate increased by two orders of magnitude and reached $10^{5}{ }^{\circ} \mathrm{C} / \mathrm{sec}$, which is due to fusion of the rod end with arc discharge. Argon was a plasma-supporting gas. The melt is removed by centrifugal forces from the rod end as $50-400 \mu \mathrm{m}$ droplets that solidify in air. The chamber is cooled down with water. $\mathrm{The} \mathrm{WC}-\mathrm{W}_{2} \mathrm{C}$ alloy powder particles are spherical.

Metallographic analysis employed optical (NEOPHOT 21) and scanning electron (REM 106I) microscopes.

X-ray diffraction studies of the phase composition, stress-strain state, size of coherent scattering domains, etc., were conducted using a RIGAKU ULTIMA IV diffractometer with different techniques, such as Rietveld and Reference Intensity Ratio (RIR) methods in $\mathrm{Cu}-K_{\alpha_{1,2}}$ radiation, $\lambda_{\mathrm{Cu}-K \alpha_{1}}=0.1541 \mathrm{~nm}$.

The stresses were determined with the $\sin ^{2} \psi$ and diffraction peak shift methods [6]. The phase composition of the alloys and the reflections on the $\theta-2 \theta$ diffraction patterns allowed us to use the $\sin ^{2} \psi$ method to locate the peaks of the same X-ray line for alloys produced by different methods.

The alloy microhardness was determined with a PMT-3 microhardness meter at a load of $150 \mathrm{~g}$ on the diamond pyramid.

\section{EXPERIMENTAL RESULTS}

X-ray diffraction has shown that the alloys solidified at all cooling rates in question mainly consist of tungsten monocarbide and semicarbide. The phase content is not dependent on the solidification rate. The main phase components, $\mathrm{WC}$ and $\mathrm{W}_{2} \mathrm{C}$, have been revealed by $\mathrm{X}$-ray diffraction of the $\mathrm{WC}-\mathrm{W}_{2} \mathrm{C}$ alloy on $\theta-2 \theta$ patterns (Fig. 1). Regardless of the cooling rate, the WC phase content is almost the same, $18-19 \mathrm{wt} . \%$, and that of the $\mathrm{W}_{2} \mathrm{C}$
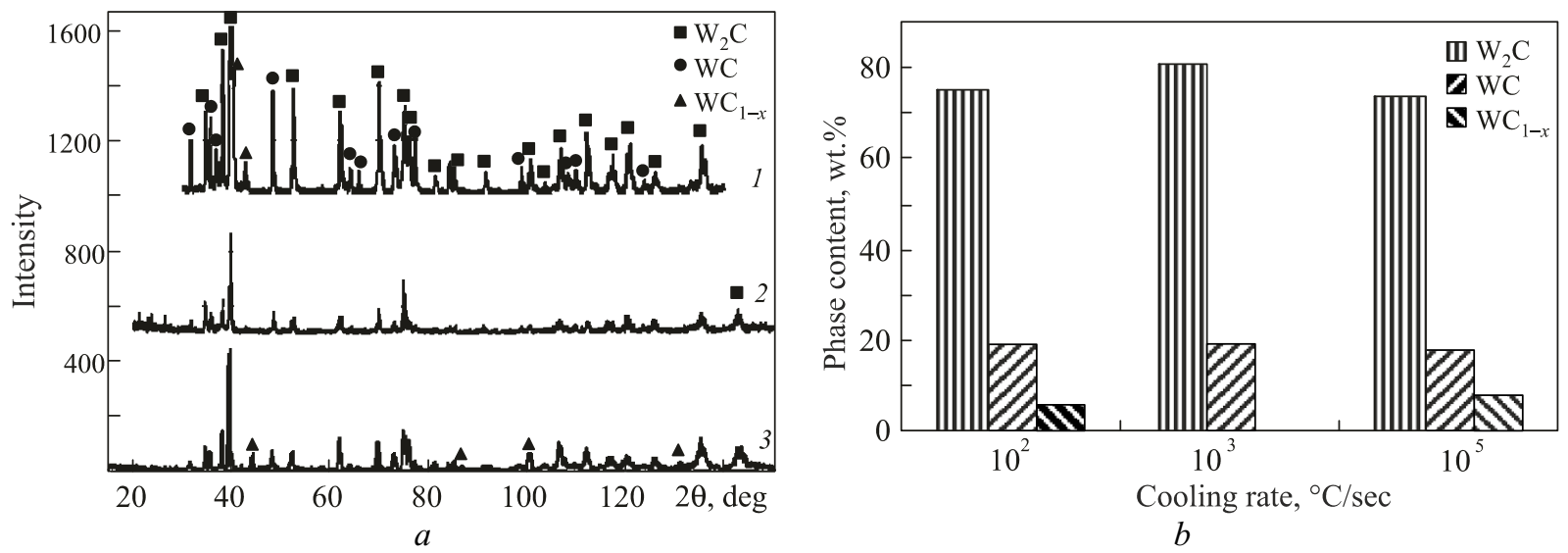

Fig. 1. X-ray diffraction patterns showing distribution of phase components $(a)$ and phase contents $(b)$ in the $\mathrm{WC}-\mathrm{W}_{2} \mathrm{C}$ alloy: cooling rate is $10^{2}(1), 10^{3}(2)$, and $10^{5}{ }^{\circ} \mathrm{C} / \mathrm{sec}(3)$ 
phase is $74-82$ wt.\%. Besides the said phases, we revealed insignificant amounts of $\mathrm{WC}_{1-x}$ both in a Tammann furnace (6 wt.\%) and during centrifugal atomization (9 wt.\%).

Electron-beam melting promoted solidification of the $\mathrm{WC}-\mathrm{W}_{2} \mathrm{C}$ alloy with the phase ratio being the closest to the stoichiometry of relit. This is associated with more complete homogenization of the melt and hence of the solidified alloy. The presence of $\mathrm{WC}_{1-x}$ in the alloys produced by centrifugal atomization and in a Tammann furnace testifies that carbon is partially lost in the solidification process.

Figure $2 a-c$ shows microstructures of the alloys produced at different cooling rates in a Tammann furnace, during electron-beam melting, and by centrifugal atomization.

The microstructures formed in a Tammann furnace are the most inhomogeneous. The microstructure is more uniform, fine-crystalline, and with elongated grains following centrifugal atomization. According to scanning electron microscopy, the alloy microstructure represents a matrix from tungsten semicarbide $\mathrm{W}_{2} \mathrm{C}$ pierced with elongated WC grains (Fig. 2).

Electron microprobe analysis has confirmed that the darker phase contains less tungsten than the light matrix phase of tungsten semicarbide. With increasing solidification rate, the tungsten carbide grains are refined in cross-section and become more elongated. At the lowest cooling rates, the WC grains are elongated, interconnected plates and rods.

When the cooling rate increases, the plates become dendritic branches, which are several times thinner than the plates and rods of tungsten monocarbide solidified at the minimal cooling rate. Solidification at the maximal cooling rate leads to a cellular structure whose dendritic grains emerge from columnar elements of regular shape with side branches that solidified at about $90^{\circ}$ and with finer, randomly placed branches of tungsten monocarbide. This structurization process suggests that increase in the solidification rate primarily changes the concentration overcooling, causing the melt to deviate from equilibrium composition according to the phase diagram. Excess
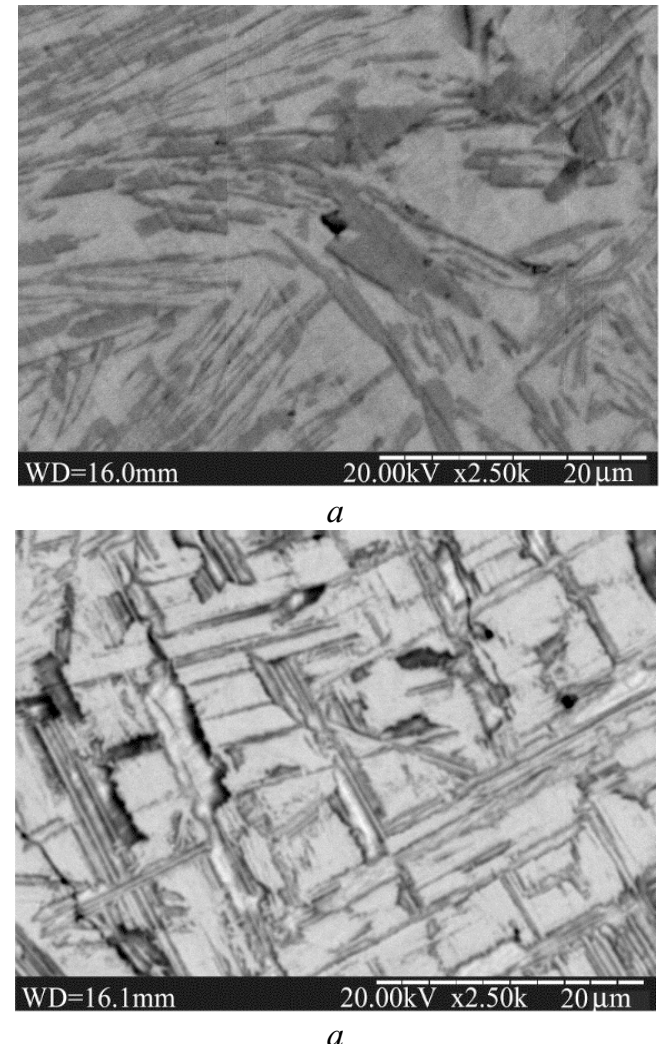
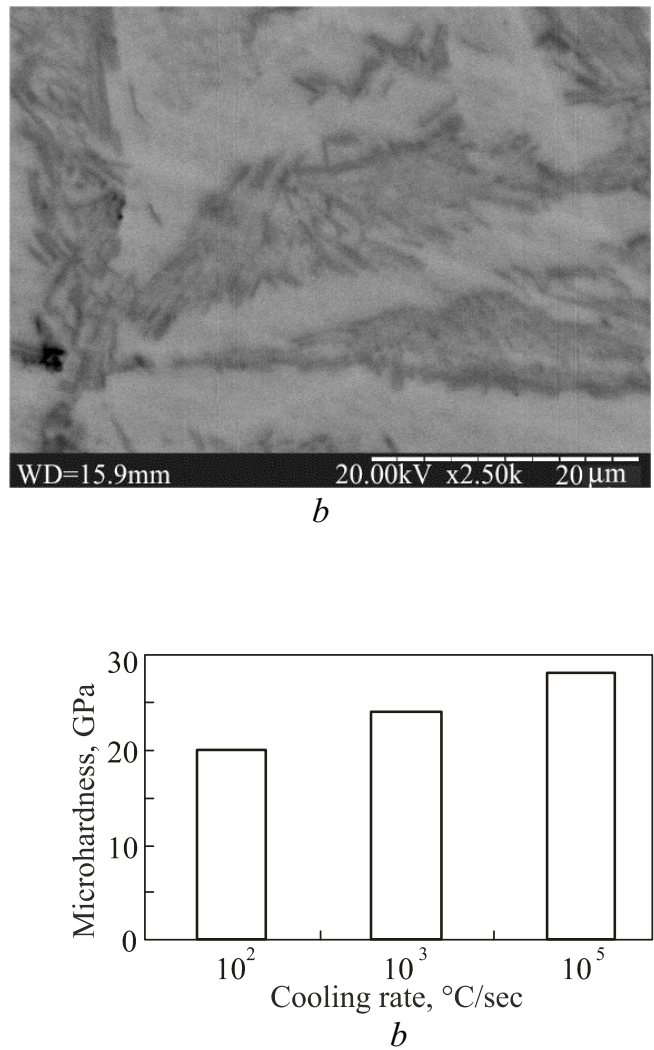

Fig. 2. Microstructure $(a-c)$ and microhardness $(d)$ of the $\mathrm{WC}-\mathrm{W}_{2} \mathrm{C}$ alloy: cooling rate is $10^{2}(a), 10^{3}$ (b), and $10^{5}{ }^{\circ} \mathrm{C} / \mathrm{sec}(c)$ 


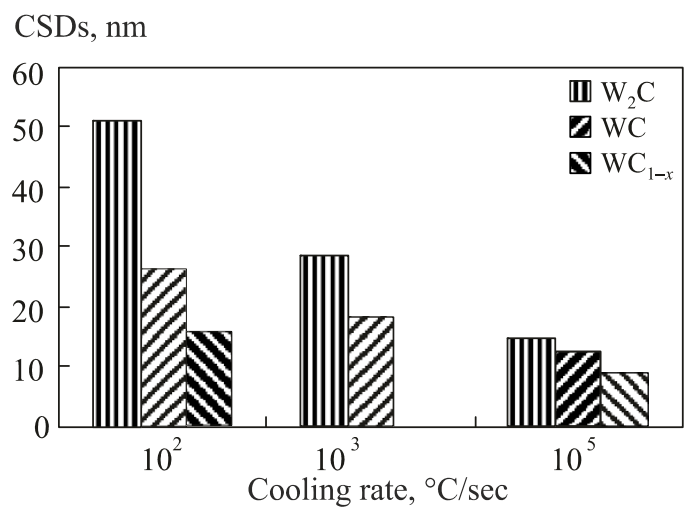

Fig. 3. Size of coherent scattering domains of $\mathrm{WC}-\mathrm{W}_{2} \mathrm{C}$ alloy phase components versus cooling rate

TABLE 1. Stresses in the WC- $\mathrm{W}_{2} \mathrm{C}$ Alloy Determined with the $\sin ^{2} \psi$ Method

\begin{tabular}{|c|c|c|}
\hline \multirow{2}{*}{ Cooling rate during solidification, ${ }^{\circ} \mathrm{C} / \mathrm{sec}$} & $\mathrm{W}_{2} \mathrm{C}$ & $\sigma_{1}+\sigma_{2}, \mathrm{GPa}$ \\
\cline { 2 - 3 } & -0.781 & $\mathrm{WC}$ \\
\hline $10^{2}$ & 0.72 & -0.820 \\
$10^{3}$ & 2.033 & -1.124 \\
$10^{5}$ & -1.479 \\
\hline
\end{tabular}

Note. $2 \theta$ and $h k l$ for $\mathrm{W}_{2} \mathrm{C}$ are $84^{\circ}$ and 202 and for $\mathrm{WC}$ are $98^{\circ}$ and 112.

carbon forms before the solidification front and leads to dendritic growth of tungsten monocarbide, which changes to cellular growth with increasing temperature gradient in the melt.

Hardness measurement has established the average integral microhardness of the alloys produced at different cooling rates (Fig. $2 d$ ). The highest microhardness $\left(28 \mathrm{GPa}\right.$ ) is observed at a cooling rate of $10^{5}{ }^{\circ} \mathrm{C} / \mathrm{sec}$. The greatest hardness and strength are known [7] to be reached by materials that represent a matrix from one refractory compound reinforced with single-crystalline fibers of another refractory compound. This mechanism takes place in the $\mathrm{WC}-\mathrm{W}_{2} \mathrm{C}$ alloy, where $\mathrm{W}_{2} \mathrm{C}$ is a matrix reinforced with fine $\mathrm{WC}$ grains.

As already noted, hardness of the $\mathrm{WC}-\mathrm{W}_{2} \mathrm{C}$ alloy depends on the cooling rate. The phase amounts remaining practically the same, the structure, size, and shape of the components change substantially. Higher cooling rates accelerate structural transformations, stimulate the formation of finer grains (Fig. 3), and influence their crystallographic orientation with respect to the direction of indentation during hardness measurement.

The hardness may also increase under mechanical stresses in phase components. Table 1 shows stresses $\left(\sigma_{1}+\sigma_{2}\right)$ in the WC- $\mathrm{W}_{2} \mathrm{C}$ alloy determined by the $\sin ^{2} \psi$ method (Fig. $4 a$ ).

The $\mathrm{W}_{2} \mathrm{C}$ and $\mathrm{WC}$ phases in the alloy produced in a Tammann furnace show tensile stresses between 0.78 and $0.82 \mathrm{GPa}$, respectively. The stresses increase during electron-beam melting and centrifugal atomization. The WC phase is elongated and the $\mathrm{W}_{2} \mathrm{C}$ phase is compressed in the alloys. The highest stresses in the two phases are observed after centrifugal atomization at the greatest cooling rate. The stresses also result in a shift of interference lines, which is the most evident in reflections at large Bragg angles.

The $\theta-2 \theta$ diffraction patterns for the $\mathrm{WC}-\mathrm{W}_{2} \mathrm{C}$ alloy produced at different cooling rates show a shift of reflections. Stresses in surface layers of the sample in the direction perpendicular to its surface $\left(\sigma_{1}+\sigma_{2}\right)$ are determined as [6]:

$$
\varepsilon=-\frac{\mu}{E}\left(\sigma_{1}+\sigma_{2}\right)
$$

where $E$ is elastic modulus (Young's modulus) and $\mu$ is Poisson's ratio. 

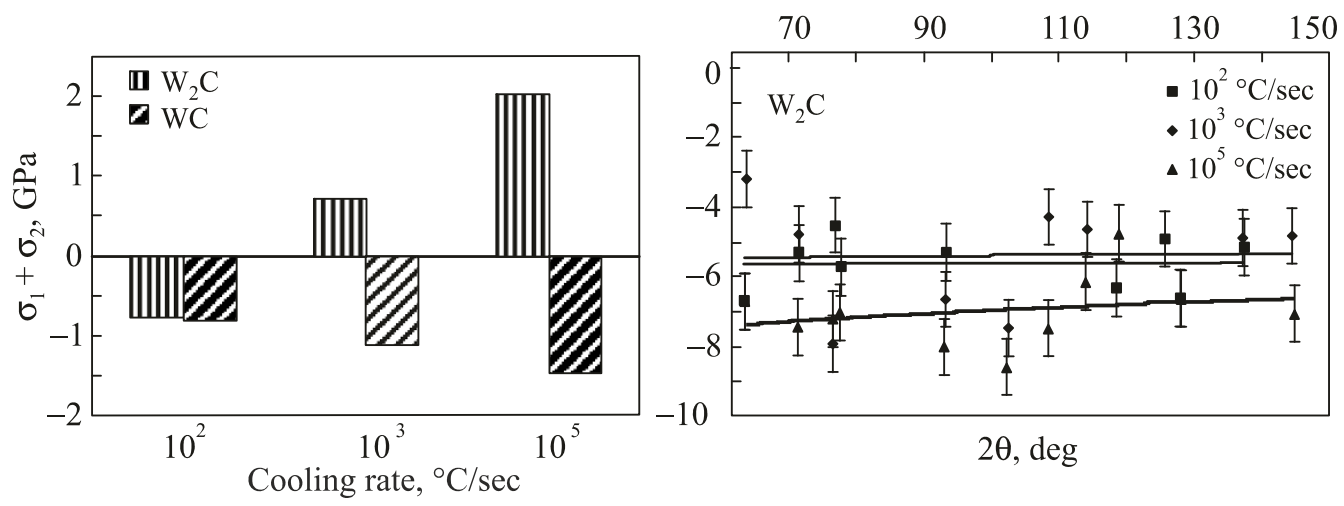

$a$

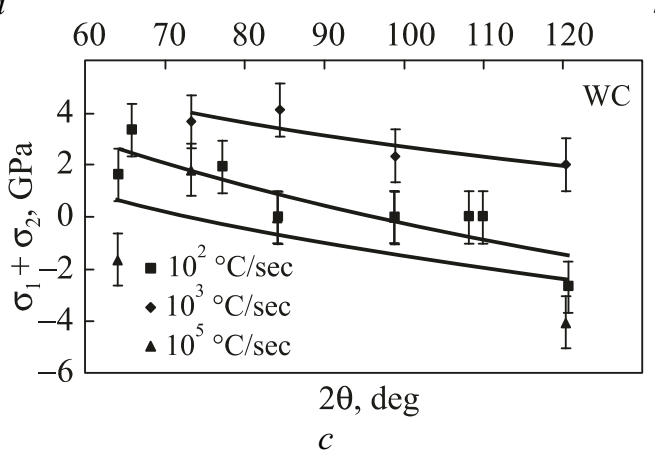

Fig. 4. Stresses in the $\mathrm{WC}-\mathrm{W}_{2} \mathrm{C}$ alloy phases determined with the $\sin ^{2} \psi$ method $(a)$ and diffraction peak shift method for $\mathrm{W}_{2} \mathrm{C}(b)$ and $\mathrm{WC}(c)$

The calculation of $\varepsilon=\Delta d / d_{\mathrm{us}}$ is based on determining the change in spacing ( $\Delta d=d_{\mathrm{us}}-d_{\mathrm{s}}$ ) between atomic planes parallel to the sample surface in unstressed $\left(d_{\mathrm{us}}\right)$ and stressed $\left(d_{\mathrm{s}}\right)$ states:

$$
\left(\sigma_{1}+\sigma_{2}\right)=-\frac{E}{\mu} \cdot \frac{\Delta d}{d_{\mathrm{us}}} .
$$

The positions of peaks on the $\mathrm{WC}-\mathrm{W}_{2} \mathrm{C}$ diffraction patterns were used to determine $d_{\mathrm{us}}, d_{\mathrm{s}}$, and $\Delta d$ and calculate $\left(\sigma_{1}+\sigma_{2}\right)$ for the alloys under study (Fig. $4 b$ and $c$ ). Stresses in the $\mathrm{W}_{2} \mathrm{C}$ phase determined from reflections in the range $2 \theta=65-145^{\circ}$ are negative (compressive) at all cooling rates. They are 5.7, 5.6, and $7 \mathrm{GPa}$ at cooling rates of $10^{2}, 10^{3}$, and $10^{5}{ }^{\circ} \mathrm{C} / \mathrm{sec}$, respectively.

Stresses in the WC phase were also determined from a series of reflections in the range $2 \theta=65-125^{\circ}$. They have the opposite sign at all cooling rates $\left(10^{2}, 10^{3}\right.$, and $\left.10^{5}{ }^{\circ} \mathrm{C} / \mathrm{sec}\right)$ and are 1,3 , and $1 \mathrm{GPa}$, respectively.

Note that alloys fail to a greater extent under tensile stresses. Compressive stresses reduce the sensitivity of materials to stress concentrators and increase their fatigue strength. Despite possible deviations in elastic modulus for reflections, the absolute values of their shift influence the stresses, which are almost the same at different lines.

Besides stresses, the shift of reflections may also result from lattice defects of the first kind [6]. This can explain the difference in stresses determined with the diffraction peak shift and $\sin ^{2} \psi$ methods.

Stresses are induced in the following conditions:

- $\quad$ plastic deformation of a polycrystalline sample through elastic and plastic anisotropy of crystals;

- $\quad$ nonuniform heating or cooling of the sample that leads to tensile and compressive stresses that are likely to induce plastic shear, causing residual stresses (they will be higher in a multiphase material if the phases have different thermal expansion coefficients); ${ }^{*}$

${ }^{*}$ The thermal expansion coefficient is $(5.2-7.3) \cdot 10^{-6}{ }^{\circ} \mathrm{C}^{-1}$ for $\mathrm{WC}$ and $(1.2-11.4) \cdot 10^{-6}{ }^{\circ} \mathrm{C}$ for $\mathrm{W}_{2} \mathrm{C}$. 
- decomposition of a supersaturated solid solution (aging) when stresses emerge in the matrix and at the boundary of the precipitating phase;

- local structural or chemical transformations involving change in the specific volume of the phases relative to the alloy.

Stresses in crystallites cause the interference lines to increase by $\Delta \beta / \beta$, where $\Delta \beta$ is deviation of broadening for a given line from $\beta$ in equilibrium solidification conditions.

\section{CONCLUSIONS}

It has been established that centrifugal atomization leads to a more homogenous fine structure with grains being uniformly distributed in the matrix of the $\mathrm{WC}-\mathrm{W}_{2} \mathrm{C}$ alloy. The alloy microstructure represents a matrix from tungsten semicarbide $\mathrm{W}_{2} \mathrm{C}$ reinforced with fine grains of tungsten monocarbide $\mathrm{WC}$.

It has been revealed that higher cooling rates increase stresses in the composite, compressive stresses being observed in the matrix phase and tensile stresses in the reinforcing phase.

It has been determined by electron microprobe analysis that microstructure is refined: coherent scattering domains decrease from 508 to $146 \mu \mathrm{m}$ for $\mathrm{W}_{2} \mathrm{C}$, from 260 to $123 \mu \mathrm{m}$ for $\mathrm{WC}$, and from 155 to $90 \mu \mathrm{m}$ for $\mathrm{WC}_{1-x}$ with increasing cooling rate.

It has been shown that microhardness of the alloys increases by a factor of 1.5 with higher cooling rates. This fact agrees with the grain refinement and change in stress-strain state of phases in the composite, which can be attributed to grain-boundary strengthening.

\section{REFERENCES}

1. P. I. Loboda, "Superhard directly reinforced composites based on refractory compounds," Metalloved. Obrab. Met., No. 1-2, 99-105 (1998).

2. I. M. Fedorchenko, I. N. Frantsevich, I. D. Radomysel'skii, et al., Powder Metallurgy, Materials, Technology, Properties, and Applications: Handbook [in Russian], Naukova Dumka, Kiev (1985), p. 624.

3. B. N. Arzamasov (ed.), V. A. Brostrem, N. A. Bushe, et al., Structural Materials: Handbook [in Russian], Mashinostroenie, Moscow (1990), p. 688.

4. A. I. Belyi, "Wear resistance and strength of tungsten carbides $\mathrm{WC}-\mathrm{W}_{2} \mathrm{C}$ produced by different methods," Avtomat. Svarka, No. 12, 20-23 (2010).

5. V. I. Dzykovich, "Properties of tungsten carbide powders produced using different processes," Avtomat. Svarka, No. 4, 28-31 (2010).

6. M. A. Krivoglaz, X-Ray and Neutron Diffraction in Imperfect Crystals [in Russian], Naukova Dumka, Kiev (1983), p. 407.

7. P. I. Loboda, Physicochemical Fundamentals for Developing New Boride Materials for Electronics and High-Performance Ceramic Cathode Assemblies [in Ukrainian], Author's Abstract of ScD Thesis, Kiev (2004), p. 29. 